\title{
ST Segment Duration Single Beat
}

National Cancer Institute

\section{Source}

National Cancer Institute. ST Segment Duration Single Beat. NCI Thesaurus. Code

C117804.

An electrocardiographic interval measured from the J point to the onset of the T wave of a single beat utilizing one or more leads. (CDISC) 